\title{
Isolated Torsion of the Fallopian Tube: a case report
}

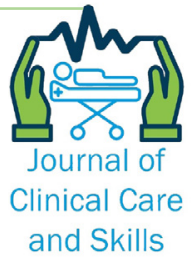

\section{ARTICLE INFO}

\section{Article Type}

Case Study

\section{Authors}

Eslamnik P.S. ${ }^{1} M D$,

Makari A. ${ }^{2} B S C$

Rezaei H. ${ }^{* 2} P h D$

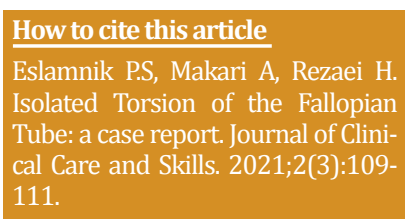

${ }^{1}$ Department of Obstetrics and Gynecology, Faculty of Medicine, Yasuj University of Medical Sciences, Yasuj, Iran ${ }^{2}$ Cellular and Molecular Research Center, Yasuj University of Medical Sciences, Yasuj, Iran

\section{*Correspondence}

Address: Shahid Montazeri St., Continuing Education Center of Yasouj University of Medical Sciences.

Phone: +98 (74) 33233024

Fax: +98 (74) 33233024

rezaie.habib1@gmail.com

\section{Article History}

Received: May 17, 2021

Accepted: July 07, 2021

ePublished: September 12, 2021

\begin{abstract}
A B S T R A C T
Aims Isolated torsion of the fallopian tube is a rare condition. A case of isolated torsion of the left fallopian tube associated without anomaly and pathology was present.

Patient \& Methods The patient was 17-year-old nulligravid from Yasuj who came to the obstetric department with acute severe abdominal pain, nausea, and vomiting in 2021. Her medical history included a left side ovarian cystectomy one year before admission. The pregnancy test was negative. Transvaginal ultrasonography showed a hemorrhagic cyst structure measuring $4 \mathrm{~cm}$ in the left adnexa with moderate free fluid in the cul-de-sac was noted; the ovaries and uterus were unremarkable. Due to acute abdominal pain, an exploratory laparotomy was done and confirmed the diagnosis of isolated torsion of the left oviduct. The ipsilateral ovary appeared normal, but the fallopian tube was gangrenous, and left salpingectomy was performed.

Findings Due to acute abdominal pain, an urgent laparotomy was performed, which revealed a twisted left fallopian tube and confirmed the isolated torsion of the left oviduct. The ipsilateral ovary appeared normal, but the fallopian tube was gangrenous, and left salpingectomy was performed, and she was discharged from our hospital two days later. Conclusion Women, without any abnormality and pathology of the tube, with a history of ovarian cyst surgery at the antimesenteric level, that the ovarian fimbria was removed are at risk for fallopian tube torsion.
\end{abstract}

Keywords Fallopian Tubes; Torsion Abnormality; Abdominal Pain; Gynecology 


\section{Introduction}

Isolated torsion of the fallopian tube is the rotation of the tube that is a rare condition. In the young adolescents and reproductive age group. Incidence was $1 / 1.5$ million ${ }^{[1-2]}$. The right tubal torsion is much more common than the left tubal torsion, which can be due to fixation of the left tube in the left hemipelvis by the sigmoid colon and mesentery [3]. It occurs without ovarian involvement. It can occur at any age but is most common at reproductive age ${ }^{[4]}$. A variety of conditions can produce tubal torsion, such as prior tubal ligation, tubal neoplasm, pelvic inflammatory disease, a long or congested mesosalpinx, para tubal tumors, trauma. Long tube length, Pyo salpinx, hydrosalpinx, ovarian cyst, para ovarian cyst, hematosalpinx, hypermobility, spasm, congenital anomalies, and ovulation induction [5]. A variety of pathologic conditions can produce a similar clinical picture and thus make diagnoses problematic such as Ectopic pregnancy, abortion, rupture of ovarian cyst, hemorrhagic cyst, ovarian torsion, pelvic infection, appendicitis, and diverticulitis [6].

Presenting symptoms of isolated torsion of the fallopian tube include acute onset of lower abdominal pain accompanied by nausea, vomiting, and peritoneal signs [7]. It is important in immediate diagnosis and treatment to maintain the fallopian tube ${ }^{[8]}$.

There are many case report about isolated tubal torsion, including Kolovos, Antoniu, Kardakis, Toyoshima, Gross, Raziel that are different from our case [3, 7, 9-11], because in this case, Isolated torsion of the left tube occurs without any abnormality and pathology. Therefore, a case of isolated torsion of the left fallopian tube associated without abnormality and pathology was present.

\section{Patient \& Methods}

The patient was a 17-year-old nulligravid from Yasuj, who came to the obstetric department with acute severe abdominal pain, nausea, and vomiting in 2021. Her medical history included a left side ovarian cystectomy one year before admission. On physical examination, tenderness was observed in her lower left quadrant, and rebound pain was marked in this area. A pelvic examination revealed an enlarged left adnexal mass. The laboratory results showed a white cell count of $16 \times 103$ cells $/ \mathrm{mm} 3$, a hemoglobin concentration of $10.0 \mathrm{~g} / \mathrm{dL}$, and a hematocrit of $30 \%$. Her pregnancy test was negative. Transvaginal ultrasonography showed a hemorrhagic cyst measuring $4 \mathrm{~cm}$ in the left adnexa with moderate free fluid in the cul-de-sac was noted; the Right ovary and uterus were normal.

\section{Findings}

Due to acute abdominal pain, an urgent laparotomy was performed, which revealed a twisted left fallopian tube and confirmed the isolated torsion of the left oviduct (Figure1). The ipsilateral ovary appeared normal (Figure 2), but the fallopian tube was gangrenous (Figure 3), and left salpingectomy was performed, and she was discharged from our hospital two days later.

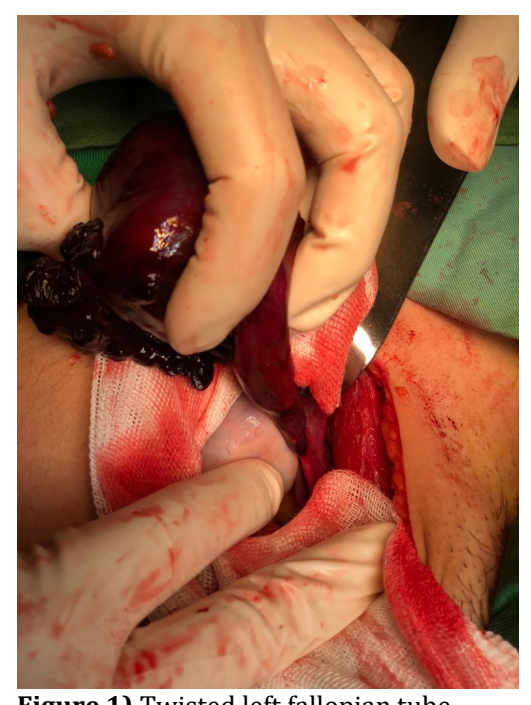

Figure 1) Twisted left fallopian tube

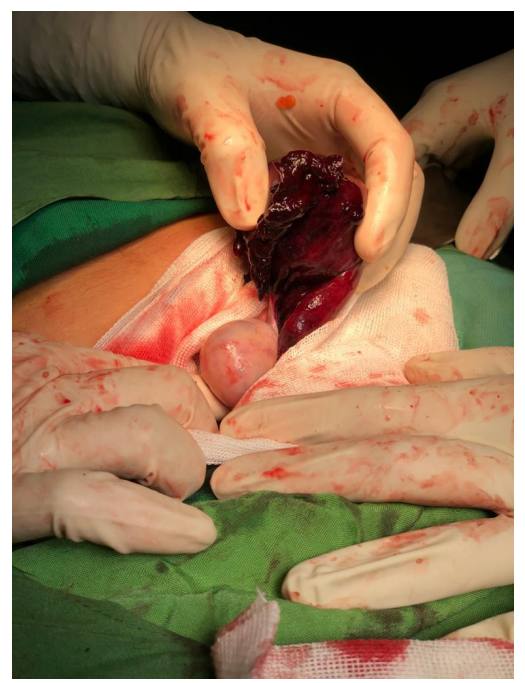

Figure 2) The ipsilateral ovary appeared normal

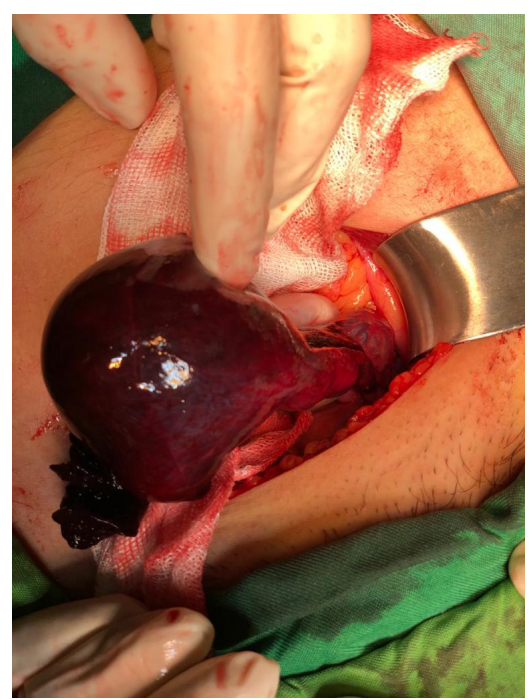

Figure 3) The fallopian tube was gangrenous 
111

\section{Discussion}

The torsion of the left tube occurs in cases with abnormality and pathologic signs such as prior tubal ligation, tubal neoplasm, pelvic inflammatory disease, a long or congested mesosalpinx, para tubal tumors, trauma. Long tube length; Pyo salpinx; hydrosalpinx; ovarian cyst; para ovarian cyst; hematosalpinx; hypermobility, spasm, congenital anomalies, and ovulation induction; pelvic adhesion [5]. We present a case of isolated torsion of the left fallopian tube associated without abnormality and pathology. In our patient, the most probable cause of isolated fallopian tubal torsion was surgery at ovarian cyst at the antimesenteric level, that the ovarian fimbria was removed. Kolovos describe a 16year-old girl with Isolated torsion of the fallopian tube with a para-ovarian cystic tumor, which was both hemorrhagically infarcted and partially gangrenous [5], but in our case, we do not have any cyst. Antoniou described a 39-year-old female whose medical history included an appendectomy and right hydrosalpinx diagnosis [9], but we do not have any hydrosalpinx in our case. Kardakis described a 38year-old null gravida woman with torsion of the right fallopian tube that Omental adhesions were presented in laparotomy at the right lower quadrant [7] but did not have any adhesion in our case. Toyoshima described a 63-year-old Japanese woman with a black necrotic, solid tumor arising from the distal end of the right fimbria. Her right fallopian tube was twisted with the tumor, but her right ovary was normal and not involved [2]. In our case, we do not have any tumors. Gross described a dilated, torsed, and necrotic right fallopian tube that was tense and darkened in appearance, measuring $8 \times 10 \mathrm{~cm}$. The ovaries were normal in appearance with Omental adhesions in the right lower quadrant [10] but did not have any adhesion in our case. Raziel reports an 18year-old woman with acute right lower quadrant pain, nausea, and vomiting. Torsion of the Fallopian tube was detected by laparoscopy, and detorsion was performed. Two years later, a second similar detorsion was performed [11] but did not have any previous torsion in our case.

One of the limitations of this study was the lack of an ultrasound image of the patient despite having a written ultrasound report. Researchers suggest surgery was done on the antimesenteric surface and don,t remove fimbria ovaries in surgery. Researchers suggest that in a future study in patients with ovarian surgery, the prevalence of fimbria ovarian injury and torsion of ovary and fimbria be evaluated.

\section{Conclusion}

Women, without any abnormality and pathology of the tube, with a history of ovarian cyst surgery at the antimesenteric level that the ovarian fimbria was removed are at risk for fallopian tube torsion.

\section{Acknowledgments: -}

Ethical Permissions: In presenting the case, attention was paid to the patient's anonymity, and informed consent was obtained from the patient to introduce the measures taken for her in the form of a case report.

Conflicts of Interests: All authors state that there is no conflict of interest.

Authors' Contribution: Eslamnik P.S. (First Author), Introduction Writer/Methodologist/Original Researcher (50\%); Makari A. (Second Author), Methodologist/Discussion Writer (20\%); Rezaei H. (Third Author) Introduction Writer/Discussion Writer (30\%)

Funding/Support: There was no funding.

\section{References}

1- Hansen $\mathrm{OH}$. Isolated torsion of the fallopian tube. Acta Obstet Gynecol Scand. 1970;49(1):3-6.

2- Toyoshima M, Mori H, Kudo K, Yodogawa Y, Sato K, Kudo $\mathrm{T}$, et al. Isolated torsion of the fallopian tube in a menopausal woman a pre-pubertal girl: Two case reports. J Med Case Rep. 2015;9:258.

3- Kolovos GN, Meytap E, Samartzis N, Kalaitzopoulos DR. Isolated torsion of the fallopian tube in a 16-year-old girl: $\mathrm{A}$ case report and review of the literature. Case Rep Womens Health. 2019;23:00132.

4- Georgios LA, Georgia SI, Matthaios TK. A clinical tool for diagnosis of isolated torsion of the right fallopian tube in a virgin girl. J Reprod Infertil. 2020;21(3):225-7.

5- Yalcin OT, Hassa H, Zeytinoglu S, Isiksoy S. Isolated torsion of fallopian tube during pregnancy; report of two cases. Eur J Obstet Gynecol Reprod Biol. 1997;74(2):17982.

6- Joshua S, Broder MD. Diagnostic imaging for the emergency physician. Amsterdam: Elsevier; 2011.

7- Kardakis S, Barranca A, Vitelli A, Amore I, Trento F, Caccia G. Isolated fallopian tube torsion. Case Rep Obstet Gynecol. 2013;2013:1-3.

8- Balasubramaniam D, Duraisamy KY, Ezhilmani M, Ravi S. Isolated fallopian tube torsion: A rare twist with a diagnostic challenge that may compromise fertility. J Hum Reprod Sci. 2020;13(2):162-7.

9- Antoniou N, Varras M, Akrivis C, Kitsiou E, Stefanaki S, Salamalekis E. Isolated torsion of the fallopian tube: A case report and review of the literature. Clin Exp Obstet Gynecol. 2004;31(3):235-8.

10- Gross M, Blumstein SL, Chow LC. Isolated fallopian tube torsion: A rare twist on a common theme. Am J Roentgenol. 2005;185(6):1590-2.

11- Raziel A, Mordechai E, Friedler S, Schachter M, Pansky $\mathrm{M}$, Ron-El R. Isolated recurrent torsion of the fallopian tube: Case report. Hum Reprod. 1999;14(12):3000-1. 